\title{
Características mecânicas e ópticas de resinas bulk-fill: revisão de literatura
}

\section{Mechanical and optics characteristics of bulk-fill resins: literature review}

Cristina Balensiefer Vicenzi*

Paula Benetti*

\section{Resumo}

Objetivo: realizar uma análise descritiva das características mecânicas e ópticas das resinas bulk-fill com base em dados da literatura. Revisão da literatura: para a realização do estudo, foram utilizados artigos extraídos das bases de dados PubMed, SciELO e Lilacs. As palavras-chave utilizadas para busca foram: resina bulk-fill, resina composta, polimerização, grau de conversão, caracterização, dureza, rugosidade, resistência à flexão, translucidez, propriedades ópticas. Os compostos bulk-fill são basicamente formados por matriz orgânica e partículas de alumínio, silício e bário, medindo de $0,1 \mu \mathrm{m}$ a $1 \mu \mathrm{m}$. Tratam-se de resinas translúcidas que podem ser polimerizadas adequadamente em camadas espessas, possuem propriedades mecânicas aceitáveis, porém inferiores às resinas nano e micro-híbridas, e têm baixo grau de contração volumétrica e menor tensão de contração do que as resinas compostas convencionais. Considerações finais: as resinas bulk-fill são indicadas para restauração de cavidades com fator de contração cavitário desfavorável. A cobertura dessas resinas com $2 \mathrm{~mm}$ de resina composta convencional em área oclusal é recomendável.

Palavras-chave: Propriedades mecânicas. Propriedades ópticas. Resina.

\section{Introdução}

A longevidade dos tratamentos restauradores diretos está relacionada, entre outros fatores, ao tipo de material empregado. Resinas compostas convencionais necessitam ser aplicadas em incrementos pequenos, para evitar tensões decorrentes da contração de polimerização e polimerização incompleta. Contudo, a técnica incremental implica sensibilidade técnica e demanda um tempo clínico elevado $^{1}$. Dessa forma, resinas compostas de baixa contração (bulk-fill) foram introduzidas no mercado, possibilitando a utilização de incrementos maiores de resina (até $4 \mathrm{~mm}$ ) para confecção de restaurações ${ }^{2}$. Entretanto, devido à grande diversidade de tipos de resina, de formas de aplicação, de propriedades mecânicas e ópticas, falta ao cirurgião-dentista subsídios para conhecer, comparar e escolher o material mais adequado para cada situação clínica.

Devido à grande disponibilidade comercial de resinas de contração mínima, há necessidade de compilação e apresentação organizada das informações presentes na literatura sobre essas resinas para possibilitar ao cirurgião-dentista a escolha do material mais adequado para cada situação clínica. Portanto, essa pesquisa apresenta e compara características das resinas bulk-fill. O trabalho tem relevância acadêmica e científica por trazer informações 
pertinentes a profissionais, pacientes e acadêmicos, especialmente quanto sobre as reais características, as possibilidades, as vantagens e as desvantagens de cada resina bulk-fill. A tomada de decisão entre as diferentes técnicas restauradoras deve ter como base o conhecimento sobre os diferentes materiais e a facilidade da técnica de aplicação.

Portanto, o objetivo da presente revisão é descrever as características mecânicas e ópticas das resinas bulk-fill, com base em dados da literatura. Especificamente, o estudo descreve e compara características das resinas bulk-fill, incluindo: composição orgânica e inorgânica, dinâmica de polimerização, grau de conversão, grau de contração, propriedades mecânicas e ópticas, variações de técnicas de aplicação e indicações clínicas.

\section{Revisão da literatura}

Para realização do estudo, foram utilizadas informações extraídas de artigos das bases de dados PubMed, SciELO e Lilacs. As seguintes palavraschave foram utilizadas: bulk fill, composite resin, polimerization, degree of conversion, characterization, hardness, roughness, flexural strength, translucency, optical properties.

\section{Resinas bulk-fill}

Restaurações diretas são as mais realizadas na odontologia. Embora o desenvolvimento de materiais ativados por luz tenha facilitado a manipulação e melhorado a qualidade das resinas compostas, alguns problemas inerentes à utilização desses materiais foram encontrados, como a tensão de contração que ocorre durante a polimerização e a subpolimerização (polimerização incompleta ou baixo grau de conversão) dos compósitos ${ }^{3}$. Para evitar problemas com a contração de polimerização, sugere-se que os materiais sejam aplicados em incrementos de até $2 \mathrm{~mm}$ de espessura. Dessa forma, seria possível minimizar a contração de polimerização ${ }^{4}$. Entretanto, a técnica é de difícil execução e demanda um tempo de trabalho maior.

Compósitos restauradores com contração de polimerização reduzida, também denominados resinas bulk-fill, têm sido desenvolvidos e estão disponíveis no mercado odontológico ${ }^{2}$. Devido à alta translucidez, eles permitem que a luz incidente penetre mais profundamente na resina, permitindo que a luz alcance profundidade de até $4 \mathrm{~mm}$ quando fotoativada por 20 segundos $^{5-7}$. $\mathrm{O}$ inovador sistema de fotoativação levou ao encurtamento do tempo de fotopolimerização e ao aumento da profundidade de polimerização.

Segundo a literatura, algumas formas de reduzir a contração encontradas nessas resinas são: incorporação de componentes capazes de interagir com o fotoiniciador e modular a cinética de polimerização, incorporação de mais de um fotoiniciador, conversão relativamente mais lenta, prolongação da fase pré-gel e fluidez e retardo da geleificação ${ }^{8,9}$.

\section{Composição das resinas bulk-fill}

Em estudo de Fronza et al. ${ }^{10}$ (2017), observou-se, microscopicamente, que o tamanho das partículas de carga inorgânica variou de $0,1 \mu \mathrm{m}$ a $1 \mu \mathrm{m}$ em diferentes formulações comerciais de compostos resinosos bulk-fill. As partículas de carga, constituídas por alumínio, silício e bário, apresentaram formas irregulares, esféricas ou cilíndricas.

Algumas das abordagens relatadas na literatura para obter maior translucidez são: diminuir a quantidade de partículas de carga e aumentar o tamanho das partículas de carga, o que resulta em menor dispersão de luz e consequente aumento da penetração de luz em profundidade ${ }^{9}$.

A composição orgânica e inorgânica de algumas resinas bulk-fill disponíveis comercialmente é apresentada no Quadro 1.

Quadro 1 - Descrição de resinas bulk-fill quanto a nome comercial, fabricante, composição da matriz orgânica, tipo e quantidade de carga inorgânica

\begin{tabular}{|c|c|c|c|c|c|}
\hline Código & Resina Composta & Fabricante & Martiz orgânica & Carga & $\begin{array}{c}\% \text { de carga em } \\
\text { Volume }\end{array}$ \\
\hline HER & Herculite Classic & $\begin{array}{l}\text { Kerr Co, Orange, CA, } \\
\text { EUA (4009366) }\end{array}$ & Bis-GMA, TEGDMA & Vidro de Borosilicato-alumínio & 59 \\
\hline SDR & Surefil SDR flow & $\begin{array}{l}\text { Dentsply Caulk, Mildford, DE, } \\
\text { EUA (08153) }\end{array}$ & $\begin{array}{l}\text { UDMA modificado, } \\
\text { TEGDMA, EBPDMA }\end{array}$ & $\begin{array}{l}\text { Vidro de Bário-alumínioflúor- } \\
\text {-borosilicato, vidro de Estrôn- } \\
\text { cio-alumínioflúor-borosilicato }\end{array}$ & 44 \\
\hline FBF & Filtek Bulk Fill & $\begin{array}{l}\text { 3M ESPE, St. Paul, MN, } \\
\text { EUA (402919) }\end{array}$ & $\begin{array}{l}\text { Bis-GMA, Bis-EMA, } \\
\text { UDMA, TEGDMA, } \\
\text { resinas Procrylat }\end{array}$ & $\begin{array}{l}\text { Zircônia/sílica, trifluoreto de } \\
\text { itérbio }\end{array}$ & 42,5 \\
\hline TEC & $\begin{array}{l}\text { Tetric EvoCeram } \\
\text { Bulk Fill }\end{array}$ & $\begin{array}{l}\text { Ivoclar Vivadent, AG, Schaan, } \\
\text { Listeinstaine (R04686) }\end{array}$ & Bis-GMA, UDMA & $\begin{array}{l}\text { Vidro de Bário, trifluoreto de } \\
\text { itérbio, óxidos e pré-polímeros }\end{array}$ & $\begin{array}{c}60 \\
\text { (17\% pré-polímeros) }\end{array}$ \\
\hline EXP & EverX Posterior & $\begin{array}{l}\text { GC Corporation, Tóquio, } \\
\text { Japão (1401152) }\end{array}$ & $\begin{array}{l}\text { Bis-GMA, TEGDMA, } \\
\text { PMMA }\end{array}$ & $\begin{array}{l}\text { Frações híbridas de carga e } \\
\text { fibras de vidro E }\end{array}$ & 57 \\
\hline
\end{tabular}




\section{Mecanismo de polimerização}

A reação de polimerização necessita que a luz utilizada seja capaz de excitar as moléculas fotoiniciadoras (geralmente as canforoquinonas). Uma vez excitadas, essas moléculas reagem com as substâncias ativadoras (aminas terciárias).

A contração gera estresse de polimerização, que pode ser influenciado por características da formulação do compósito, como tipo de matriz, conteúdo de carga ${ }^{1,11}$, cinética de polimerização, grau de conversão, módulo de elasticidade ${ }^{11,12}$ e geometria da cavidade $^{12,13}$. Várias tentativas têm sido feitas para minimizar os efeitos negativos da polimerização, como técnica de inserção incremental ${ }^{11}$, introdução de um monômero à base de silorano ${ }^{14}$ e mudanças na composição da matriz e fotoiniciador ${ }^{1,8}$.

As resinas bulk-fill utilizadas no estudo de Tsujimoto et al. ${ }^{16}$ (2017) continham um modulador de polimerização de alto peso molecular para reduzir a contração volumétrica ${ }^{15}$. Essa única estrutura molecular contribui para o atraso do ponto gel, o que representa um aumento da viscosidade por meio da formação de cadeia, podendo permitir mais tempo para compensar o encolhimento e, então, reduzir a contração volumétrica ${ }^{5}$. Por esse motivo, o tipo de monômero da resina pode ser um fator mais influenciador do que o conteúdo de carga ${ }^{16}$.

Um estudo recente de Kumagai et al. ${ }^{17}$ (2015) demonstrou que os valores de força de união em paredes gengivais são significativamente maiores quando um composto bulk-fill (SureFil SDR Flow, Dentsply) é utilizado em comparação com composto convencional nanoparticulado (Filtek Z350, Oral Care), independentemente de ser inserido pela técnica incremental ou pela de preenchimento.

\section{Grau de conversão}

Vários sistemas iniciadores incorporados nas resinas bulk-fill podem melhorar a profundidade de polimerização ${ }^{18}$. Com maior translucidez, as resinas bulk-fill permitem que a luz penetre profundamente, o que leva a uma maior polimerização dos monômeros $^{8}$.

Uma grande profundidade de polimerização em resinas bulk-fill pode ser explicada devido à presença do fotoiniciador Ivocerin, derivado do dibenzoílo germânio, associado com canforoquinona/sistema iniciador amino. Esse iniciador é ativado pela luz ultravioleta $(380-450 \mathrm{~nm})$, sendo gerador de radicais livres mais eficiente que a canforoquinona, levando a uma rápida polimerização e a uma alta conversão monomérica ${ }^{19}$.

Segundo instruções dos fabricantes das resinas SureFil SDR Flow e Tetric EvoCeram, 20 segundos de fotoativação são suficientes para polimerizar um incremento de $4 \mathrm{~mm}$. Porém, 40 segundos ou mais parece ser mais adequado para um incremento de $4 \mathrm{~mm}^{16}$.

\section{Propriedades mecânicas e ópticas}

Novos materiais têm demonstrado propriedades mecânicas excelentes (Venus Bulk Fill, Tetric EvoCeram Bulk-Fill RBC, X-tra base hybrid RBC, X-tra fill hybrid RBC, SonicFill ${ }^{\mathrm{TM}}$ Nanohybrid RBC, Filtek ${ }^{\mathrm{TM}}$ Bulk Fill nano RBC, e Xenius e Coltene Dual-Cure Bulk-Fill RBCs) ${ }^{15,20}$. Entretanto, as propriedades mecânicas desses materiais têm se mostrado menos satisfatórias do que as de resinas nano-híbridas e micro-híbridas ${ }^{21}$.

Partículas de carga com diâmetro que se aproxima da metade do comprimento de onda da luz utilizada para fotoativação aumentam a dispersão de luz. Portanto, a transmissão de luz tende a reduzir com o aumento do tamanho de partículas e sua irregularidade na forma ${ }^{10}$. Grande quantidade de carga tende a reduzir a transmissão de luz, devido à diferença de índice de refração de luz na interface entre as partículas de carga e a resina ${ }^{22}$.

Um estudo comparativo de transmissão de luz entre resinas nano-híbridas, flow e bulk-fill demonstrou que os compósitos bulk-fill utilizados no estudo apresentaram maior translucidez do que resinas convencionais ${ }^{9}$. Portanto, a profundidade de conversão depende de composição monomérica, concentração de iniciador, forma e translucidez do material e fonte de irradiação de luz ${ }^{23}$.

De acordo com os fabricantes, a resina bulk-fill não requer uma camada de cobertura superficial adicional de resina composta e pode ser usada como material de preenchimento em único passo. Entretanto, estudos comprovam que a maioria dos materiais bulk-fill demonstraram propriedades mecânicas menores, se comparados a compostos resinosos convencionais, e seu uso em locais de alta carga oclusal deve ser cauteloso ${ }^{15}$.

Na Tabela 1, apresenta-se uma síntese dos principais achados dos estudos sobre propriedades das resinas bulk-fill. 
Tabela 1 - Valores médios para propriedades mecânicas e ópticas de resinas bulk-fill comercialmente disponíveis

\begin{tabular}{|c|c|c|c|c|c|c|}
\hline Estudo & Resina (Fabricante) & Microdureza & $\begin{array}{l}\text { Grau de } \\
\text { Conversão } \\
(\%)\end{array}$ & $\begin{array}{l}\text { Profundidade de } \\
\text { polimerização* } \\
(\mathrm{mm})\end{array}$ & $\begin{array}{l}\text { Contração } \\
\text { volumétrica } \\
(\%)\end{array}$ & $\begin{array}{l}\text { Resistência } \\
\text { à flexão } \\
(\mathrm{MPa})\end{array}$ \\
\hline Czasch e Ilie ${ }^{27}$ (2013) & $\begin{array}{l}\text { Surefil SDR Flow (Dentsply) } \\
\text { Venus Bulk Fill (Heraeus Kulzer) }\end{array}$ & $\begin{array}{l}59.8 \\
46.4\end{array}$ & $\begin{array}{l}59.7 \\
66.1\end{array}$ & & & \\
\hline Jang et al..$^{5}(2014)$ & $\begin{array}{l}\text { Surefil SDR Flow (Dentsply) } \\
\text { Venus Bulk Fill (Heraeus Kulzer) } \\
\text { Tetric N-Ceram Bulk Fill (Ivoclar) }\end{array}$ & $\begin{array}{l}32.14 \\
30.55 \\
49.05\end{array}$ & & & $\begin{array}{l}25.36 \\
32.14 \\
11.57\end{array}$ & \\
\hline AlQahtani et al. ${ }^{28}$ (2015) & Tetric Evo Ceram (Ivoclar) & & & 3.5 & & \\
\hline Al-Ahdal et al. ${ }^{29}$ (2015) & $\begin{array}{l}\text { X-tra base (Voco) } \\
\text { Venus Bulk Fill (Heraeus Kulzer) } \\
\text { Tetric EvoCeram Bulk Fill (Ivoclar) } \\
\text { Sonic Fill (Kerr Corp.) } \\
\text { Filtek Bulk Fill (3M ESPE) } \\
\text { Restorative Bulk Fill ever XPosterior } \\
\text { (GC Corporation) } \\
\text { Beautiful-Bulk Flowable (Shofu) } \\
\text { Beautiful-Bulk Restorative (Shofu) } \\
\end{array}$ & & $\begin{array}{l}57.6 \\
74.8 \\
53.0 \\
70.2 \\
62.3 \\
59.9 \\
\\
58.4 \\
47.6\end{array}$ & & & \\
\hline Kemaloglu et al. ${ }^{30}(2015)$ & Filtek Bulk Fill (3M ESPE) & 817.10 & & & & \\
\hline Algamaiah et al. ${ }^{31}$ (2016) & $\begin{array}{l}\text { SureFil SDR Flow (Dentsply) } \\
\text { Tetric EvoCeram Bulk Fill (Ivoclar) } \\
\text { Filtek Bulk Fill Flowable Restorative } \\
\text { (3M Oral Care) } \\
\end{array}$ & & & & $\begin{array}{l}3.65 \\
2.44 \\
3.47\end{array}$ & \\
\hline Alkhudhairy ${ }^{25}$ (2017) & $\begin{array}{l}\text { Filtek Bulk Fill (3M ESPE) } \\
\text { Tetric N-Ceram (Ivoclar) } \\
\text { SonicFill (Kerr) } \\
\text { Surefil SDR Flow (Dentsply) }\end{array}$ & $\begin{array}{l}46.36 \\
39.23 \\
58.26 \\
27.27\end{array}$ & & & & $\begin{array}{l}235.48 \\
224.06 \\
262.64 \\
253.19\end{array}$ \\
\hline Fronza et al. ${ }^{10}(2017)$ & $\begin{array}{l}\text { Surefil SDR Flow (Dentsply) } \\
\text { Filtek Bulk Fill (3M ESPE) } \\
\text { Tetric Evo Ceram (Ivoclar) } \\
\text { EverX Posterior (GC Corporation) } \\
\end{array}$ & & & & & $\begin{array}{c}146 \\
143.2 \\
76.2 \\
104.2\end{array}$ \\
\hline Jung e Park² (2017) & $\begin{array}{l}\text { Surefil SDR Flow (Dentsply) } \\
\text { Venus Bulk Fill (Heraeus Kulzer) } \\
\text { Tetric N-Ceram Bulk Fill (Ivoclar) } \\
\text { Sonic Fill (Kerr) }\end{array}$ & $\begin{array}{l}32.1 \\
25.2 \\
49.3 \\
70.5\end{array}$ & & & $\begin{array}{l}32.38 \mu \mathrm{m} \\
34.33 \mu \mathrm{m} \\
15.31 \mu \mathrm{m} \\
16.43 \mu \mathrm{m}\end{array}$ & $\begin{array}{c}101.26 \\
97.36 \\
100.21 \\
136.67\end{array}$ \\
\hline Moharam et al..$^{33}$ (2017) & $\begin{array}{l}\text { X-tra Fil (Voco GmbH) } \\
\text { Sonic-Fill (Kerr Corporation) }\end{array}$ & $\begin{array}{l}95.16 \\
89.01\end{array}$ & & $\begin{array}{l}0.976 \\
0.904\end{array}$ & & \\
\hline Rodriguez et al..$^{34}$ (2017) & $\begin{array}{l}\text { Sonic Fill (Kerr) } \\
\text { Tetric EvoCeram Bulk Fill (Ivoclar) }\end{array}$ & & & $\begin{array}{l}0.82 \\
0.73 \\
\end{array}$ & & \\
\hline Tsujimoto et al. ${ }^{16}$ (2017) & $\begin{array}{l}\text { SDR (Dentsply) } \\
\text { Filtek Bulk Flow (3M ESPE) } \\
\text { Tetric Evo Ceram Bulk Fill (Ivoclar) } \\
\text { Filtek Bulk Fill (3M ESPE) }\end{array}$ & & & $\begin{array}{l}3.91 \\
3.73 \\
3.86 \\
3.57\end{array}$ & $\begin{array}{l}2.11 \\
2.10 \\
2.15 \\
1.95\end{array}$ & $\begin{array}{l}116.8 \\
120.9 \\
130.5 \\
129.5\end{array}$ \\
\hline
\end{tabular}

* Valores para 20 segundos de fotoativação.

Fonte: elaboração dos autores.

\section{Discussão}

A composição inorgânica básica da maioria dos compostos resinosos de incremento único (bulk-fill) compreende a matriz resinosa monomérica e partículas de carga: alumínio, silício e bário ${ }^{10}$.

Compostos bulk-fill são resinas translúcidas que permitem que a luz incidente penetre mais profundamente na resina, em camadas espessas, alcançando profundidade de até $4 \mathrm{~mm}$, quando fotoativada por 20 segundos $^{5-7}$, com propriedades mecânicas aceitáveis e baixo grau de contração de polimerização, o que resulta em menor tensão de contração do que as resinas compostas convencionais ${ }^{9}$.

Uma dispersão de luz pode levar a uma diminuição da translucidez quando há maior quantidade de partículas de carga ${ }^{24}$. A transmissão de luz tende a reduzir com o aumento do tamanho das partículas de carga e sua irregularidade na forma ${ }^{10}$. Grande quantidade de carga tende a reduzir a transmissão de luz, devido à diferença de índice de refração de luz na interface entre as partículas de carga e a re$\operatorname{sina}^{22}$. 
Portanto, para uma maior translucidez desse material, foi preciso diminuir a quantidade de partículas de carga e aumentar o tamanho dessas partículas ${ }^{9}$. Microscopicamente, o tamanho das partículas de carga inorgânica dos compostos bulk-fill varia de $0,1 \mu \mathrm{m}$ a $1 \mu \mathrm{m}^{10}$.

Com maior translucidez, as resinas bulk-fill permitem que a luz da fotoativação penetre em profundidade, o que leva a uma maior conversão dos monômeros em polímeros ${ }^{8}$. Fotoiniciadores desenvolvidos recentemente, incluindo óxido de trimetilbenzoil difenilfosfina e um derivado de dibenzoil-germânio, foram introduzidos como intensificadores devido às suas habilidades de polimerização mais profunda e controle da duração de polimerização ${ }^{19}$.

Algumas resinas possuem um sistema adicional de fotoativação (Ivocerin), que tem demonstrado ser superior em profundidade de polimerização ${ }^{19}$. Essas resinas apresentaram baixa viscosidade devido à presença de pré-polímeros em sua composição inorgânica e à presença de fotoiniciadores como o Ivocerin, derivado do dibenzoílo germânio, que exibiram valores de resistência à flexão biaxial uniformes em profundidades de até $4 \mathrm{~mm}^{10,19}$.

O tempo de polimerização possivelmente afeta as propriedades dos materiais bulk-fill ${ }^{25}$. Uma taxa de polimerização mais lenta, conseguida por novos fotoiniciadores, diminui a tensão de contração em $60 \%$ a $70 \%^{8}$ e a contração de polimerização em $20 \%$, em comparação com as resinas compostas convencionais.

A contração gera tensão de polimerização, que pode ser influenciada por características da formulação do compósito, como tipo de matriz, conteúdo de carga ${ }^{1,11}$, cinética de polimerização, grau de conversão, módulo de elasticidade ${ }^{12,19}$ e geometria da cavidade $^{12,13}$. Várias tentativas têm sido feitas para minimizar os efeitos negativos da contração de polimerização, como técnica de inserção incremental ${ }^{11}$, introdução de um monômero à base de silorano ${ }^{14} \mathrm{e}$ mudanças na composição da matriz e do fotoiniciador ${ }^{1,8}$.

Um modulador de polimerização de alto peso molecular adicionado às resinas bulk-fill favorece a redução da contração volumétrica ${ }^{15,26}$.

As resinas bulk-fill apresentam valores de resistência de união à dentina maior do que resinas convencionais, possivelmente pela menor contração de polimerização ${ }^{17}$. Então, as resinas bulk-fill são indicadas para uso em restaurações classe I e classe II (dentes posteriores) como base ou forramento, regularização da parede pulpar e caixa proximal.

Embora novos materiais (Venus Bulk Fill, Tetric EvoCeram Bulk-Fill RBC, X-tra base hybrid RBC, X-tra fill hybrid RBC, SonicFill ${ }^{\mathrm{TM}}$ Nanohybrid RBC, Filtek ${ }^{\mathrm{TM}}$ Bulk Fill nano RBC, e Xenius e Coltene Dual-Cure Bulk-Fill RBCs) demonstrem propriedades mecânicas excelentes ${ }^{15,20}$, há estudos que mostram que são menos satisfatórias do que resinas nano-híbridas e micro-híbridas ${ }^{21}$.
Portanto, uma camada de resina convencional de $2 \mathrm{~mm}$ na oclusal é indicada quando se restaura com uma resina bulk-fill, especialmente na consistência fluida. Por ser um material fluido (menos carga), suas propriedades mecânicas são inferiores, fazendo com que o desafio da mastigação seja mais deletério. Ainda, por sua natureza fluida, a resina não permitiria escultura funcional e estética da região oclusal.

Em uma situação clínica, as resinas bulk-fill são indicadas para restauração de cavidades com fator de configuração (Fator C) desfavorável (classes I e II) e para reestabelecer contatos proximais, pois, nesses casos, a mudança volumétrica poderia comprometer o contato com o dente adjacente, deixando um espaço que poderia resultar em impacção alimentar.

\section{Considerações finais}

Compostos bulk-fill são resinas translúcidas que podem ser fotoativadas adequadamente em camadas espessas (até $4 \mathrm{~mm}$ ), com propriedades mecânicas aceitáveis e baixo grau de contração de polimerização. Uma translucidez maior permite que a luz penetre mais profundamente na resina, levando a uma polimerização maior dos monômeros.

Quando há maior quantidade de partículas de carga, a transmissão de luz tende a ser reduzida, devido à diferença de índice de refração de luz na interface entre as partículas de carga e a resina. Portanto, a carga em maior tamanho e menor quantidade é a principal responsável pela alta translucidez do compósito. O tamanho das partículas de carga das resinas bulk-fill varia de $0,1 \mu \mathrm{m}$ a $1 \mu \mathrm{m}$, e sua composição inorgânica básica é alumínio, silício e bário. Há redução da contração de polimerização em até $20 \%$ e da tensão de polimerização em até $70 \%$. A tensão de polimerização pode ser influenciada por tipo de matriz, conteúdo de carga, cinética de polimerização, grau de conversão, módulo de elasticidade e geometria da cavidade.

As canforoquinonas são as moléculas fotoiniciadoras mais utilizadas e reagem com as substâncias ativadoras (aminas terciárias). Materiais com profundidade de polimerização superior possuem um sistema adicional (Ivocerin) de fotoativação, levando a uma rápida polimerização e a uma alta conversão monomérica. Uma taxa de polimerização mais lenta diminui a contração de polimerização e, consequentemente, contribui para melhor tensão de contração.

O tipo de monômero da resina pode ser um fator mais relevante para a diminuição da tensão de contração do que o conteúdo de carga. Materiais bulk-fill fluidos apresentam maior contração do que materiais bulk-fill regulares. Resinas bulk-fill apresentam valores de resistência de união à dentina maior do que resinas convencionais. 
Fabricantes recomendam que uma fotoativação de 20 segundos é suficiente para um incremento de $4 \mathrm{~mm}$, embora estudos mostrem que 40 segundos seriam mais adequados (maior grau de conversão em grande profundidade).

As propriedades mecânicas desses materiais têm se mostrado menos satisfatórias do que as de resinas nano-híbridas e micro-híbridas. Embora a maioria dos fabricantes afirmem que a resina bulk-fill não requer uma camada superficial adicional de resina convencional, é recomendado uma camada de $2 \mathrm{~mm}$ dessa resina em áreas de alta carga oclusal.

\section{Abstract}

Objective: the objective of this work was, based on literature data, to describe mechanical and optical characteristics of bulk-fill resins. Literature review: articles were selected at PubMed, SciELO and Lilacs. Search was performed using the keywords: bulk-fill resin, composite resin, polymerization, degree of conversion, characterization, hardness, roughness, flexural strength, translucency, optical properties. Bulk-fill composites are basically formed by organic matrix and particles of aluminium, silicon and barium, measuring from $0.1 \mu \mathrm{m}$ to $1 \mu \mathrm{m}$. They are translucent resins that can be polymerized in thick layers, with acceptable mechanical properties, but with inferior performance than nano and microhybrid resins. The material presents volumetric lower shrinkage and residual stresses than conventional composite resins. Final considerations: bulk-fill resins are indicated for the restoration of cavities with an unfavorable contraction factor. A 2 mm-thick layer of conventional composite resin at the occlusal area over the bulk-fill resins is recommended.

Keywords: Mechanical properties. Optics properties. Resin.

\section{Referências}

1. Braga RR, Ballester RY, Ferracane JL. Factors involved in the development of polymerization shrinkage stress in resin-composites: a systematic review. Dent Mater 2005; 21(10):962-70.

2. Fráter M, Forster A, Keresztúri M, Braunitzer G, Nagy K. In vitro fracture resistance of molar teeth restored with a short fiber-reinforced composite material. J Dent 2014; 42:1143-50.

3. Ferracane JL, Greener EH. The effect of resin formulation on the degree of conversion and mechanical properties of dental restorative resins. J Biomed Mater Res 1986; 20(1):121-31.

4. Anusavice KJ. Phillips materiais dentários. 11. ed. Rio de Janeiro: Elsevier; 2005

5. Jang JH, Park SH, Hwang IN. Polymerization Shrinkage and Depth of cure of Bulk-Fill Resin Composites and Highly Filled Flowable Resin. Oper Dent 2014; 39(6).

6. Orlowski M, Tarczydlo B, Chalas R. Evaluation of marginal integrity of four bulk-fill dental composite materials: in vitro study. Sci World J 2015; 2015.
7. Tarle TA, Marovic D, Andermatt L, Ristic M, Taubock T. Influence of irradiation time on subsurface degree of conversion and microhardness of high-viscosity bulk-fill resin composites. Clin Oral Investig 2015; 19:831-40.

8. Ilie N, Hickel R. Investigations on a methacrylate-based flowable composite based on the SDR technology. Dent Mater 2011; 27(4):348-55.

9. Bucuta S, Ilie N. Light transmittance and micro-mechanical properties of bulk-fill vs. conventional resin based composites. Clin Oral Investig 2014; 18(8)1991-2000.

10. Fronza BM, Ayres APA, Pacheco RR, Rueggeberg FA, Dias CTS, Giannini M. Characterization of inorganic filler contente, mechanical properties, and light transmission of bulk-fill resin composites. Oper Dent 2017; 42(4):445-55.

11. Poggio C, Chiesa M, Scribante A, Mekler J, Colombo M. Microleakage in Class II composite restorations with margins below the CEJ: in vitro evaluation of diferente restorative techniques. Med Oral Patol Cir Bucal 2013; 18(5):793-8.

12. Tantbirojn D, Pfeifer CS, Braga RR, Versluis A. Do low-shrink composites reduces polymerization shrinkage effects. J Dent Res 2011; 90:569-601.

13. Hofmann N, Hunecke A. Influence of curing methods and matrix type on the marginal seal of class II resin-based composite restorations in vitro. Oper Dent 2006; 31:97-105.

14. Karaman E, Ozgunaltay G. Polymerization shrinkage of different types of composite resins and microleakage with and without liner in class II cavities. Oper Dent 2014; 39:325-31.

15. Leprince JG, Palin WM, Vanacker J, Sabbagh J, Devaux J, Leloup G. Physico-mechanical characteristics of commercially available bulk- fill composites. J Dent 2014; 42(8):993-1000.

16. Tsujimoto A, Barkmeier WW, Takamizawa T, Latta MA, Miyazaki M. Depth of cure, flexural properties and volumetric shrinkage of low and high viscosity bulk-fill giomers and resin composites. Dent Mater J 2017; 36(2):205-13.

17. Kumagai RY, Zeidan LC, Rodrigues JA, Reis AF, Roulet JFJ. Bond Strenght of a Flowable Bulk-fill Resin Composite in Class II MOD cavities. Adhes Dent 2015; 17(5):427-32.

18. Palin WM, Senyilmaz DP, Marquis PM, Shortall AC. Cure width potential for MOD resin composite molar restorations. Dent Mater 2008; 24(8):1083-94.

19. Moszner N, Fischer UK, Ganster B, Liska R, Rheinberger V. Benzoyl germanium derivates as novel visible light photoinitiators for dental materials. Dent Mater 2008; 24(7):901-7.

20. Li X, Pongprueksa P, Van Meerbeek B, De Munck J. Curing profile of bulk-fill resin-based composites. J Dent 2015; 43(6):664-72.

21. Abouelleil H, Pradelle N, Villat C, Attik N, Colon P, Grosgogeat B. Comparison of mechanical properties of a new fiber reinforced composite and bulk filling composites. Rest Dent End 2015; 40(4):262-70.

22. Leprince JG, Palin WM, Hadis MA, Devaux J, Leloup G. Progress in dimethacrylate-based dental composite technology and curing efficiency. Dent Mater 2013; 29(2):139-56.

23. Le Bell AM, Tanner J, Lassila LV, Kangasniemi I, Vallittu PK. Depth of light-initiated polymerization of glass fiber-reinforced composite in a simulated root canal. Int J Prosthodont 2003; 16(4):403-8.

24. Miyagawa Y, Powers JM, O'Brien WJ. Optical properties of direct restorative materials. J Dent Res 1981; 60:890-4.

25. Alkhudhairy FI. The effect of curing intensity on mechanical properties of different bulk-fill composite resins. Clin Cosmet Investig Dent 2017; 9:1-67. 
26. Zorzin J, Maier E, Harre S, Fey T, Belli R, Lohbauer U, et al. Bulk-fill resin composites: polymerization properties and extended light curing. Dent Mater 2015; 31:293-301.

27. Czasch P, Ilie N. In vitro comparison of mechanical properties and degree of cure of bulk fill composites. Clin Oral Investig 2013; 17(1):227-35.

28. Alqahtani MQ, Michaud PL, Sullivan B, Labrie D, Alshaafi MM, Price RB. Effect of high irradiance on depth of cure of a conventional and a bulk fill resin-based composite. Oper Dent 2015; 40(5).

29. Al-Ahdal K, Ilie N, Silikas N, Watts DC. Polymerization kinetics and impact of post polymerization on the Degree of Conversion of bulk-fill resin-composite at clinically relevant depth. Dent Mater 2015; 31(10):1207-13.

30. Kemaloglu H, Kaval ME, Turkun M, Kurt SM. Effect of novel restoration techniques on the fracture resistance of teeth treated endodontically: an in vitro study. Dent Mater 2015; 34(5):618-22.

31. Algamaiah H, Sampaio CS, Rigo LC, Janal MN, Giannini M, Bonfante EA, et al. Microcomputed tomography evaluation of volumetric shrinkage of bulk-fill composites in class II cavities. J Esthet Restor Dent 2016; 29(2):118-27.

32. Jung JH, Park SH. Comparison of polymerization shrinkage, physical properties, and marginal adaptation of flowable and restorative bulk-fill resin-based composites. Oper Dent 2017; 42(4):375-86

33. Moharam LM, El-Hoshy AZ, Abou-Elenein K. The effect of different insertion techniques on the depth of cure and vickers surface micro-hardness of two bulk-fill resin composite materials. J Clin Exp Dent 2017; 9(2):266-71.

34. Rodriguez A, Yaman P, Dennison J, Garcia D. Effect of light-curing exposure time, shade, and thickness on the depth of cure of bulk fill composites. Oper Dent 2017; 42(3):505-13.

\section{Endereço para correspondência:}

Cristina Balensiefer Vicenzi

Avenida Sete de Setembro, 51, apto 303, Centro

99010-120, Passo Fundo, RS, Brasil

Telefone: (54) 981118223

E-mail: crisvicenzi@gmail.com;

paulabenetti@upf.br

Recebido: 28/02/18. Aceito: 19/03/18. 